\title{
Amino acids as energy sources
}

\section{By D. B. Lindsay, Biochemistry Department, ARC Institute of Animal Physiology, Babraham, Cambridge $C B 24 A T$}

To discuss amino acids as energy sources seems at first sight a rather bizarre notion. It is not that there are any particular difficulties in accepting the concept, but rather that it seems a somewhat extravagant or whimsical idea, rather like a discussion of caviar as a pig food. Nevertheless, the topic does merit discussion. For some animals protein regularly predominates in the diet. In adult humans in the Western world protein intake is normally substantially greater than the requirement for amino acids, so the excess is simply of value as an energy source. In addition, a knowledge of factors that determine the catabolism of amino acids might enable us to minimize these losses when the availability of amino acids is limited.

It is proposed to consider the catabolism of amino acids firstly with respect to a supply of dietary amino acids, and secondly with respect to endogenously derived amino acids.

\section{Dietary amino acids}

The general principles underlying the catabolism of the indispensable amino acids are well illustrated by some recent studies of Kang-Lee \& Harper (1977; 1978). They examined the effect in rats, of variation in dietary intake of histidine or threonine on the rate of catabolism. With a low intake of these amino acids, the plasma concentrations remained low and little of the $\left({ }^{14} \mathrm{C}\right.$-labelled) dietary amino acid appeared as ${ }^{14} \mathrm{CO}_{2}$. When the intake was greater than the dietary requirement, there was a sharp rise in the plasma amino acid concentration and a marked rise in the rate of ${ }^{14} \mathrm{CO}_{2}$ production. These observations are readily explained on biochemical grounds. Enzymes initiating the catabolism of amino acids have Michaelis constants in the milli-molar range (Krebs, 1972) while for enzymes initiating protein synthesis (e.g. t-RNA synthetases of threonine; Allende et al. 1966) and arginine (Allende \& Allende, 1964) the values are in the micromolar range. Thus at low concentration, amino acids will be largely bound to the synthetases. Only when the synthetic pathway of protein is saturated will the catabolic pathway assume significant importance. Increasing the dietary intake of threonine or histidine does not induce increased catabolic enzyme activity, but this occurs following a high protein diet. In these circumstances there is still no increased oxidation when the diet is switched to one limiting for threonine or histidine, but when they are present in an amount above requirement, the increased enzyme activity results in an increased rate of oxidation.

\section{Endogenous amino acids}

Sites of catabolism. Miller (1962) showed that although most amino acids are catabolized in the liver, the branched-chain and some dispensable amino acids $\infty 29-6651 / 80 / 3913-3402$ Sor.00 (c) 1980 The Nutrition Society 
could also be catabolized in peripheral tissues. These results have been supported by studies with isolated muscle (Manchester, 1965; Goldberg \& Odessey, 1972; Beatty et al. 1974) in which only the branched-chain acids and aspartate, glutamate and alanine were shown to be oxidized to any appreciable extent. These experiments, in which recovery of ${ }^{14} \mathrm{CO}_{2}$ from ${ }^{14} \mathrm{C}$-labelled amino acids were measured, are a necessary, but not sufficient condition for demonstration of oxidation, since ${ }^{14} \mathrm{CO}_{2}$ may arise from exchange reactions in the tricarboxylic cycle. Chang \& Goldberg (1978b) have indeed argued that much ${ }^{14} \mathrm{CO}_{2}$ in these studies arises in this way, and that apart from leucine, there is only a modest amount of oxidation in muscle. Indeed, Hutson et al. (1978) have suggested that for the branched-chain acids, while the first (transamination) reaction occurs in muscle, the ketoacids so formed are decarboxylated relatively slowly in muscle, and that much further oxidation may occur in the liver.

The plausibility of the view that in sheep the liver is the main site of catabolism has been tested for five indispensable amino acids; threonine, lysine and methionine, and the branched-chain acids, leucine and isoleucine. Estimates of hepatic uptake have been obtained from the results of Wolff et al. (1972) and Lindsay et al. (1975) as well as some unpublished results of J. L. Mangan and F. A. Harrison and D. W. Pethick and D. B. Lindsay, which are essentially confirmatory. These results show that even with substantial variation in conditions there is only moderate variation in hepatic uptake, so that the range of values obtained may be related to other studies in which the (whole animal) oxidation of these amino acids has been measured (Brookes et al. 1973; Morton et al. 1978; Egan \& MacRae, 1979; Gill \& Ulyatt, I979; J. C. Mackenzie and D. B. Lindsay, unpublished results). For threonine, lysine and methionine, even if it is assumed that all the oxidation that is observed occurs in the liver, the uptake is sufficient to account for substantial rates of hepatic protein synthesis-up to $30-35 \mathrm{~g} / \mathrm{d}$. For isoleucine and leucine, however, if the oxidation occurred in the liver, there would not be sufficient amino acid taken up to meet the needs of protein synthesis. Indeed even if no oxidation of the branched-chain acids occurred in the liver, the uptake would in general be only sufficient to account for protein synthesis of approximately $20 \mathrm{~g} / \mathrm{d}$.

In contrast, Miss Mackenzie in my laboratory has demonstrated that ${ }^{14} \mathrm{CO}_{2}$ is produced from $\left[\mathrm{I}^{14} \mathrm{C}\right]$ leucine in sheep hind-limb muscles in vivo. The evidence, however, does not really support the view that muscle is the major site for the oxidation of leucine. With plausible assumptions as to the total muscle mass, and the extent to which the muscle studied is representative, it is likely that less than one-third of the total leucine oxidation occurs in muscle. There is other evidence that the capacity to oxidize leucine may be found in many tissues. Odessey \& Goldberg (1972) have shown that isolated tissue from brain, kidney and adipose tissue of rats is active in this respect. Wohlt et al. (1977) have shown that the uptake of the branched-chain acids by the bovine mammary gland is much more than is needed for protein synthesis, and that isolated mammary tissue has the capacity to oxidize these acids. On the other hand, measurement of the uptake of 
amino acids by the pregnant uterus of sheep (P. J. Buttery, J. C. Mackenzie and D. B. Lindsay, unpublished results) does not suggest that uptake of the branchedchain acids is much more than is required for protein synthesis. These findings are not at variance with the now widely held view that there is significant amino acid oxidation by the foetal lamb-they merely suggest that the branched-chain acids are not oxidized to a greater extent than other indispensable amino acids.

It seems to be necessary to establish the extent of oxidation of an amino acid by studies in vivo since Noda \& Ichihara (1976) have shown that with rat kidney, for example, the capacity in vitro to oxidize amino acids bears little relation to the extent to which oxidation occurs in vivo.

Amino acid interconversions. There is a net output of amino acids from muscle in fasting man (Felig et al. 1970), sheep (Ballard et al. 1976; Lindsay et al. 1977), cattle (Bell et al. 1975) and from the perfused rat hindquarters (Ruderman \& Berger, 1974). Release does not, however, simply involve proteolysis in muscle since the proportions of amino acids released do not reflect the composition of muscle protein. In particular there is a much larger amount of alanine and glutamine and a much smaller release of aspartate and glutamate than would be expected, and in man and the rat a smaller release of the branched-chain acids. These observations have been explained (e.g. Chang \& Goldberg, 1978a) by supposing that the branched-chain acids are partly oxidized, the amino groups being transferred, via glutamate to pyruvate. Although it is generally accepted that the amino groups of alanine and glutamine are at least in part derived from other amino acids, the origin of the carbon has proved much more controversial. One view (see Chang \& Goldberg, 1978a) is that alanine-C is derived almost entirely from carbohydrate. There is an alternative view, however (see e.g. Snell, 1979), that a significant amount of alanine- $\mathrm{C}$ is derived from other amino acids. Most of the detailed arguments relate to studies in vitro with diaphragm or rat skeletal muscle, and are concerned with the relative availability of glucose or amino acid carbon, and the effects of inhibitors (see Lindsay \& Buttery, 1980). Some alanine could be derived directly from the alanine in muscle protein-it is estimated this may constitute about $25-30 \%$ of the total output. There is also no doubt that some is derived from glucose, since when $\left[{ }^{14} \mathrm{C}\right.$ glucose is added to isolated muscle preparations (Grubb, 1976), or infused in whole sheep (Heitmann et al. 1973; Chandrasena, 1976) significant labelling is demonstrable in circulating alanine. Thus the dispute is about whether $30-40 \%$ of alanine is derived from amino acids, or from muscle glycogen. Apart from the evidence referred to, it is striking that, in healthy man, exercise increases alanine release from muscle; but this does not occur in patients who lack muscle glycogen phosphorylase. Furthermore, in the plasma of eviscerated-nephrectomized rats, it is glutamine not alanine that accumulates in plasma, following loading with branched-chain amino acids (Aikawa et al. 1973).

It may be observed that even if amino acid carbon does make some contribution to alanine put out from muscle, it would seem that the only amino acids that can make a contribution are aspartate, glutamate and the branched-chain acids (apart from leucine which is not glycogenic). This is because these acids are the only ones 
for which there is some evidence of oxidation in muscle. They are therefore the only ones for which some flow through the tricarboxylic cycle intermediates is certain, and thus probably the only ones which may contribute to the intracellular pyruvate pool. If they do contribute to alanine, it should be noted that they must contribute to a greater extent to lactate output, since lactate and alanine are derived from a common intracellular pyruvate pool and the greatest fraction of the output of this pool is in the form of lactate.

The question remains as to the fate of the aspartate, glutamate, isoleucine and valine released in muscle from proteolysis. Although in labelled form they can give rise to ${ }^{14} \mathrm{CO}_{2}$ they can only suffer net oxidation provided they are first converted to acetyl $\mathrm{CoA}$ - which would appear to mean that they must first pass through the intracellular pyruvate pool. In studies with isolated muscle, Chang \& Goldberg ( $1978 b$ ) suggested that only a minor fraction of the flow of these acids passes through the pyruvate pool, and much more was found in glutamate. They suggest therefore that the major fate of these acids was conversion to glutamine. As pointed out above, the study in vivo in rats by Aikawa et al. (1973) is consistant with this finding; moreover Heitmann et al. (1973) found that in contrast to alanine, there was no labelling in plasma glutamate in sheep following infusion of $\left[{ }^{14} \mathrm{C}\right]$ glucose.

The picture that emerges then is one in which most amino acids produced from proteolysis are released from muscle unchanged; but there is oxidation of leucine and to a lesser degree of the other branched-chain acids, and aspartate and glutamate. Much of the carbon is transferred in the form of glutamine, and perhaps to a small extent as alanine; the extra nitrogen, however, is distributed between alanine and glutamine. This pattern is fairly general. There is, however, indication that oxidation of the branched-chain acids occurs to a smaller extent in ruminants. There is also some indication in sheep at least, that there is an increased output of glycine, but the origin of this is as yet not clear.

Gluconeogenesis from amino acids. It is clear that much catabolism of amino acids occurs in the liver. One problem that has proved difficult to assess quantitatively is the extent to which catabolism occurs through gluconeogenesis. We can set upper limits in fasted animals from the urinary nitrogen output; it is also possible to assess the contribution indirectly, i.e. assume that what is not accounted for when the contribution of other precursors has been assessed must be due to amino acids. These approaches in studies of sheep have been discussed elsewhere (Lindsay, 1978; 1980). Here we may briefly consider the so-called direct approach, that is, by infusion of ${ }^{14} \mathrm{C}$-labelled amino acids, and estimation of the value of glucose:precursor specific radioactivity. This approach also has difficulties because of (a) the 'exchange' reactions that occur in the tricarboxylic acid cycle and (b) the effect of hepatic protein synthesis and degradation, which results in elevated concentrations of intracellular amino acids. This renders uncertain the specific activity of the precursor amino acid. With this approach, only two amino acids appear to make a significant contribution to glucose synthesis; alanine, for which the contribution is about $5-7 \%$ of glucose synthesized, at least in adult 
sheep (Wolff \& Bergman, 1972) and pregnant sheep (D. B. Lindsay, G. J. Faichney and J. C. Mackenzie, unpublished results) and glutamine, about $4-6 \%$ of glucose synthesized (Heitmann \& Bergman, 1978). In contrast, Wolff \& Bergman (1972) found the contribution of some other dispensable amino acids (serine, aspartate and glycine) was $\mathrm{r} \%$ or less of glucose synthesized for each. For threonine (Morton et al. 1978; Egan \& MacRae, 1979) and isoleucine and methionine (Egan \& MacRae, 1979) the fractional contribution was even smaller; less than $0.5 \%$. It should be noted that for serine, glycine and threonine, the metabolic pathway for conversion to glucose probably does not involve flow through tricarboxylic cycle intermediates, and thus there is unlikely to be a significant underestimation of gluconeogenic capacity through 'exchange' reactions (Lindsay, 1978). Although there are one or two glycogenic amino acids that have not yet been studied, it seems improbable that more than about $20 \%$ of the glucose synthesized is derived from amino acids, although this figure could be appreciably increased if account is taken of exchange reactions. Perhaps more relevant in the present context is the proportion of the amino acid flux that is converted to glucose. For the indispensable amino acids only about 1 , or at most $2-3 \%$ of the flux (or about $10 \%$ of the total catabolized) passes through glucose. For the dispensable amino acids the amount is rather larger; approximately $10-25 \%$ of the flux. Even so, it would seem that conversion to glucose is not the major catabolic fate for any of the amino acids. Because of the uncertainties discussed earlier, it is desirable to estimate the maximum possible gluconeogenic contribution by an alternative procedure-from the hepatic uptake. This need not be corrected for hepatic protein synthesis, since if the liver weight is constant, there will be matching rates of synthesis and degradation. It should be corrected, however, for the 'export' proteins; albumin, transferrin, fibrinogen and lipoprotein. A rough guess would suggest that total protein secreted is about $12 \mathrm{~g} / \mathrm{d}$ in sheep. If hepatic amino acid uptake is corrected accordingly, the maximum production of glucose in sheep would be between $24-30 \mathrm{~g} / \mathrm{d}$. This would be reduced to the extent that amino acid is oxidized to $\mathrm{CO}_{2}$. This estimate is larger than is obtained by other methods, but the precision is such that it is impossible to say whether this shows that there is substantial oxidation of amino acids in the liver. It would seem to the author that this is likely, however, since in experiments with the perfused sheep liver when large amounts of amino acids, particularly alanine and glutamine, are made available, there is a large uptake, a marked stimulation of oxygen consumption but not appreciable increase in glucose output.

More than half the estimated glucose output is derived from just two amino acids-alanine and glutamine. If alanine- $\mathrm{C}$ is derived from glucose then alanine must be supposed to play a role analogous to that of lactate, that is, it is predominantly concerned with the recycling of carbohydrate- $C$ rather than the catabolism of amino acids. Although glutamine-C is probably not directly derived from glucose, it is possible that the glutamate and aspartate of peripheral protein from which it is derived, may be, at least in part, derived from plasma glucose. Thus a significant part of the glucose produced from amino acids may be concerned with 
$\mathrm{C}$ recycling rather than the catabolism of amino acids.

How far these findings in sheep may apply more generally is not certain. Alanine is a major source of glucose in man (Chochinov et al. 1978). Glutamine, however, appears to be less important both in the rat (Aikawa et al. 1973) and in man (Felig et al. 1973), although a significant part is taken up by the gut with release of alanine. It is possible therefore that in non-ruminants as in sheep, an appreciable part of gluconeogenesis is concerned with carbohydrate recycling rather than as a route for the catabolism of amino acids.

A final point for discussion is whether the catabolism of amino acids affects their anabolic use. It has been proposed that leucine has a specific effect in regulating protein synthesis in muscle (Buse \& Reid, 1975; Faulks et al. 1975; Buse et al. 1979). It is also the amino acid for which there is strongest evidence for oxidation in muscle. In several conditions there is both a decrease in rate of protein synthesis and increase in rate of leucine oxidation. This is seen in fasting (Goldberg \& Odessey, 1972) and in diabetic rats (Odessey et al. 1974) and also in haemorrhagic shock induced in rabbits (Ryan et al. 1974). The crucial question, as yet unanswered, is whether an increase in the oxidation of leucine controls or is controlled by the rate of protein synthesis.

\section{REFERENCES}

Aikawa, T., Matsutaka, H., Yamamoto, H., Okudo, T., Ishikawa, E., Kawano, T. \& Natsumura, E. (1973). J. Biochem., Tokyo 74, 1003.

Allende, C. C. \& Allende, J. E. (1964). F. biol. Chem. 239, 102.

Allende, C. C., Allende, J. E., Gatica, M., Celia, J., Mora, G. \& Matamala, M. (1966). f. biol. Chem. 241, 2245.

Ballard, F. J., Filsell, O. H. \& Jarrett, I. G. (1976). Metabolism 25, 415.

Beatty, C. H., Curtis, S., Young, M. K. \& Bocek, R. M. (1974). Am. f. Physiol. 227, 268.

Bell, A. W., Gardner, J. W., Manson, W. \& Thompson, G. E. (1 975). Br. Y. Nutr. 33, 207.

Brookes, I. M., Owens, F. N., Brown, R. E. \& Garrigus, U. S. (1973). F. Anim. Sci. 36, 965.

Buse, M. G., Atwell, R. \& Moncusi, V. (1979). Horm. Metab. Res. I I, 289.

Buse, M. G. \& Reid, S. S. (1975). J. clin. Invest. 56, 1250.

Chandrasena, L. (1976). Aspects of lactate metabolism in sheep. PhD Thesis, University of Liverpool.

Chang, T. W. \& Goldberg, A. L. (1978a). F. bio. Chem. 253, 3677.

Chang, T. W. \& Goldberg, A. L. (1978b). F. biol. Chem. 253, 3685 .

Chochinov, R. H., Perlman, K. \& Moorhouse, M. D. (1978). Diabetes 27, 287.

Egan, A. R. \& MacRae, J. C. (1979). Ann. Rech. Vet. 10, 376.

Felig, P., Wahren, J., Karl, I., Cerasi, E., Luft, R. \& Kipnis, D. M. (1973). Diabetes 22, 573.

Felig, P., Pozefsky, T., Marliss, E. \& Cahill, G. F. (1970). Science, N.Y. 167, 1003.

Fulks, R. M., Le Jeanne, B. \& Goldberg, A. L. (I975). F. bio. Chem. 250, 290.

Gill, M. \& Ulyatt, M. J. (1979). Br. F. Nutr. 41, 605.

Goldberg, A. L. \& Odessey, R. (1972). Am. F. Physiol. 223, I384.

Grubb, B. (1976). Am. F. Physiol. 230, 1379.

Heitmann, R. N. \& Bergman, E. N. (1978). Am. Y. Physiol. 234, E197.

Heitmann, R. N., Hoover, W. H. \& Sniffen, C. J. (1973). F. Nutr. 103, 1587.

Hutson, Susan M., Cree, T. C. \& Harper, A. E. (1978). F. biol. Chem. 253, 8126.

Kang-Lee, Y. A. \& Harper, A. E. (1977). J. Nutr. 107, 1427.

Kang-Lee, Y. A. \& Harper, A. E. (1978). f. Nutr. 108, 163.

Krebs, H. A. (1972). Adv. Enz. Reg. 10, 397.

Lindsay, D. B. (1978). Biochem. Soc. Trans. 6, 1152. 
Lindsay, D. B. (1979). ARC Seminar. Protein metabolism in the ruminant. [P. J. Buttery, editor]. London: Agricultural Research Council.

Lindsay, D. B. \& Buttery, P. J. (1980). In Protein Deposition in Animals. [P. J. Buttery and D. B. Lindsay, editors]. London: Butterworths.

Lindsay, D. B., Jarrett, I. G., Mangan, J. L. \& Linzell, J. L. (1975). Q. Yl. exp. Physiol. 60, 141.

Lindsay, D. B., Steel, J. W. \& Buttery, P. J. (1977). Proc. Nutr. Soc. 36, 33A.

Manchester, K. L. (1965). Biochim. biophys. Acta 100, 295.

Miller, L. L. (1962). In Amino Acid Pools p. 708, [J. T. Holden, editor]. Amsterdam: Elsevier.

Morton, J. L., Lindsay, D. B. \& Buttery, P. J. (1978). Proc. Nutr. Soc. 37, 7 A.

Noda, C. \& Ichihara, A. (1976). J. Biochem., Tokyo 80, II 59.

Odessey, R. \& Goldberg, A. L. (1972). Am. F. Physiol. 223, 1376.

Odessey, R., Khairallan, E. \& Goldberg, A. L. (1974). F. biol. Chem. 249, 7623.

Ruderman, N. B. \& Berger, M. (1974). F. biol. Chem. 249, 5500.

Ryan, N. T., George, B. C., Odessey, R. \& Egdahl, R. H. (1974). Metabolism 23, 901.

Snell, K. (1979). Trends in Biochemical Science 4, 124.

Wohlt, J. E., Clark, J. H., Derrig, R. G. \& Davis, C. L. (1977). F. Dairy Sci. 60, 1875.

Wolf, J. E. \& Bergman, E. N. (1972). Am. F. Physiol. 223, 455.

Wolf,, J. E., Bergman, E. N. \& Williams, H. H. (r972). Am. Y. Physiol. 223, $43^{8}$. 